Aus der chirurgischen Klinik in Würzburg. (Vorstand: Geheimer Hofrat Prof. Dr. F. König.)

\title{
Über die Ileusfälle der Kriegsjahre in der chirurgischen Universitätsklinik Würzburg.
}

\author{
Von Dr. Hugo Reusch.
}

Während der langen Kriegsjahre haben sich die in der Würzburger Universitätsklinik (chirurgische Abteilung) zugehenden Fälle von Ileus um einen hohen Prozentsatz vermehrt. Der Begriff Ileus ist im wesentlichen ein klinischer Sammelbegriff, der eine große Menge verschiedenartiger Krankheitsformen deckt und jeder Untersucher ist bemüht, ihn im einzelnen Falle durch einen exakteren pathologisch-anatomischen Begriff $\mathrm{zu}$ ersetzen oder - mit anderen Worten ausgedrückt - die Ursache des Ileus festzustellen. Um ein klares Bild zu erhalten, sollen nun gleich von vornherein die verschiedenen Formen des lleus festgelegt werden. Je nach Art des Hindernisses, das den Darmverschluß bedingt, wird unterschieden:

I. Der dynamische Ileus, der wieder zerfällt in:

I. Den paralytischen Ilcus, bei welchem durch traumatisch entzündliche Lähmung der Muskulatur, z. B. nach operativen Eingriffen (postoperativer Ileus) oder durch reflektorische Lähmungen bei Pankreasblutungen, Gallenstein- und Nierensteinkoliken oder durch zirkulatorisch bedingte Lähmungen (Embolic der Arteria meseraica) der Darmverschluß herbeigeführt wird.

2. Den spastischen lleus, der auf spastische Kontraktionen um einen Fremdkörper, wie Gallensteine usw., zurückgeführt wird.

II. Der mechanische Ileus, der durch viele Ursachen bedingt sein kann und der viel häufiger vorkommt, als der dynamische Ileus. Ihn kann man in folgende Unterabteilungen zerlegen:

r. Der Okklusionsilcus, z. B. bei angeborenem Darmverschlub an irgendeiner Stelle. 
2. Der Obturationsileus, bei welchem die Passagestörung durch gestielte Geschwülste des Darmes, durch Gallensteine, verhärtete Kotmassen, Fremdkörper, Würmer, durch Invagination (Invaginationsileus) hervorgerufen sein kann.

3. Der Kompressionsileus, bei welchem sich je nach Art des komprimierenden Agens wieder die verschiedensten Unterabtei. lungen ergeben, z. B. der mesenteriale DuodenalverschluB, die Kompression durch den schwangeren Uterus, durch Eierstockgeschwülste usw.

4. Der Stenosen- oder Strikturenileus, durch zirrhotische Karzinome des Darmes oder Peritoneums, durch verheilende U1. cera des Darmes, z. B. auch durch Invaginationen bedingt.

5. Der Inflektionsileus, durch Abknickungen des Darmes in folge von postoperativen oder sonstigen Verwachsungen (Adhäsionsileus), durch Strangwirkungen (Stranginflektionsileus =-Strangobturationsileus von Wilms), durch Abknickung bei zu langen Schlingenbildungen (Hirschsprungsche Krankheit) oder durch Ventilverschluß hervorgerufen.

6. Der Strangulationsileus bei Inkarzerationen, Volvulus, Knotenbildungen, WringverschluB.

Kurze Auszüge aus den Krankengeschichten der Ileusfälle der Kriegsjahre sollen uns nun in folgenden Zeilen über die Art der Darmverschlüsse, Alter und Körperbeschaffenheit der Pa. tienten usw. Aufschluß geben.

I914:

Fall I. D. D., 40jährige Baucrsfrau, mittelgroB, kräftig, in gutem Ernährungszustand.

Untersuchungsbefund: Von der Mittellinie handbreit oberhalb der Symphyse zicht ein druckempfindlicher, regelmäßiger Strang nach oben.

Operationsbefund: Adhäsionsstreifen von $2-3 \mathrm{~cm}$ Dicke von der Gegend der rechten Bruchpforte nach oben zu einigen leicht verwachsenen Dünndarmschlingen. Durch den freien Raum zwischen Strang und vorderer Bauchwand ist cine Ileumschlinge geschlüpft und abgeklemmt. Ileumschlinge hat keinen Abschnürungsring. Der Strang wird abgetragen. Geheilt entlassen.

Fall 2. B. Chr., 28 Jahre alt, mittelgroße Frau. In der recht $n$ Bauchgegend ist ein größerer Tumor zu fühlen nach oben bis zur Nabelhöhe; Darmsteifung, Kotbrechen, Windverhalten, Aufstoßen. Rektal nihil. 
Operationsbefund: Eine Bride, von der Tube ausgehend, schnürt eine Ileumschlinge dicht oberhalb der Cökalklappe ein. Die Bride wird gelöst, die Schnürfurche übernäht. Geheilt entlassen.

Fall 3. B. D., 59 Jahre alte Maurerswitwe, mittelgroß, leidend aussehend, guter Ernährungszustand. In der rechten Unterleibsgegend fühlt man einen kindskopfgroßen Tumor; Anfälle von Darmsteifung.

Operationsbefund: Der Tumor besteht aus einem Stück Netz, das durch den Inguinalkanal prolabiert ist; gleichzeitig mit dem Netz ist der Darm verwachsen und es ist deshalb zu einer erschwerten Darmpassage gekommen. Der Tumor und ein etwa $30 \mathrm{~cm}$ langes Stück lleum wird abgetragen, Darm Seite an Seite anasto. mosiert. Exitus letalis.

Fall 4. G. Ph., I8jährige Bauerstochter, mittelgroß, ziemlich kräftig, in gutem Ernährungszustand. Abdomen ist aufgetrieben, auf der linken Seite des Abdomens sieht man cine stärkere Vorwölbung und fühlt daselbst eine vermehrte Resistenz.

Operationsbefund: Das Colon ascendens ist in der Länge von ungefähr $30 \mathrm{~cm}$ vom blinden Ende aus nach links herübergeschlagen und ist dabei um seine Hauptachse gedreht. Das Mesocolon ist sehr lang und ermöglicht die Bildung eines Coccum mobile und diesen Volvulus. Der Dickdarm ist oberhalb der Abschnürungsstelle stark gebläht. Die involvierten Darmstellen sind stark injiziert und die Venen zum Teil thrombosiert.

Operation: Das Colon ascendens wird in der Höhe von $56 \mathrm{~cm}$ reseziert dicht unterhalb der Flexura hepatica. Enteroanasto. mose des Dünndarms mit dem Colon transversum. Geheilt entlassen.

F a ll 5. H. B., 44jährige Tagelöhnersgattin; guter Ernährungszustand; Schmerzen im Unterleib, kein Stuhl und Flatus, Erbrechen.

Unt ersuch ungs bef und: Leib ziemlich aufgetrieben, druckempfindlich; sichtbare Peristaltik des Colon transversum.

Operation: Eine große Schlinge des Colon transversum stark gebläht. Colon descendens und Flexura sigmoidea und Rectum gefüllt mit steinhartem Kot.

Therapie: Auf Öleinläufe entleeren sich reichlich hartc Kotmassen. Geheilt entlassen.

F all 6. Sch. K., Iojähriger Knabe, Invaginatio colica.

F all 7. W. B., Frau, Strangileus, Dünndarmresektion.

F a 11 8. H. K., Hirschsprungsche Krankheit.

F all 9. Tr. S., Ileus (Bride).

Fall Io. S. S., Ileus (Bride).

1915:

Fall II. J. L., 72jährige Frau, klein, blaß, von mäßig gutem Ernährungszustand. Plötzlich heftiges Unwohlsein, kein Abgang von Winden mehr, Aufstoßen und Erbrechen, keine Schmerzen. 
Untersuchungsbefund: Die rechte Flexur weist einen kleinen, derben lumor auf von etwas höckeriger Obcrfläche.

Behandlung: Auf hohen Einlauf entleeren sich reichlich Stuhl und Winde. Von einer Operation wird wegen der hinfälligen Konstitution der Patientin Abstand genommen. Die Patientin wird beschwerdefrei, jedoch in mattem, hinfälligem Zustande entlassen.

Fall 12. W. M., zljährige Frau, klein, mager, blaß. Patientin ist an äuberst heftigen Durchfällen erkrankt; kein Erbrechen, öfters Aufstoßen; keine Leibschmerzen. An die Durchfälle schlob sich Stuhlverhaltung an, der Leib wurde aufgetrieben.

Untersuchungsbefund: Abdomen stark aufgetrieben, Darmperistaltik nicht nachweisbar; lauter, tympanitischer Schall über dem ganzen Abdomen mit Ausnahme der abhängenden Partien, besonders rechts. Hier ist bis zu $1 / 4$ der Nabelhöhe seitlich Dämpfung nachzuweisen, die sich verkleinert, wenn Patient seitlich links liegt. Hohe Einläufe ohne Resultat. Exitus letalis.

Diag nose: Peritonitis, ileus.

Obduktionsbefund: Ileus infolge Zug und Druck des prolabierten Uterus. Diffusc, eitrig fibrinöse Peritonitis.

Fall I3. K. Th., I4jährige Tagelöhnerstochter, guter Ernährungszustand. Plötzlich Erbrechen und Leibschmerzen in der linken Unterbauchgegend.

Untersuchungsbefund: In der linken Unterbauchgegend etwas vermehrte Spannung, aber kcine deutliche Resistenz, mäbige Druckempfindlichkeit; rektal viel Scybala. Erbrechen von galligen Massen, von Speiseresten, Stuhl- und Windverhaltung.

Operation: Dünner, kräftiger Strang, unter welchen eine $30 \mathrm{~cm}$ lange Dünndarmschlinge geschlüpft war und dadurch komprimiert wurde. Nach Lösen des Stranges füllt sich der Darm, dennoch Enteroanastomose. Geheilt entlassen.

Fal1 14. F. E., 54jährige Bauersfrau von schlechtem Aussehen. An heftigen Leibschmerzen, Stuhl- und Windverhaltung, galligem Erbrechen erkrankt.

Untersuchungsbefund: Abdomen aufgetrieben, Darmsteifung. In der rechten Femoralgegend eine Hernie von Eigröße, die leicht reponibel erscheint und nicht schmerzhaft ist; links Andeutung einer Inguinalhernie. Rektale Untersuchung ohne Frgebnis.

Operation: Dünndarmschlingen zum Teil stark gebläht, zum Teil eng zusammengefallen. Die Einklemmungserscheinungen werden hervorgebracht durch Verschlingung einer Dünndarmschlinge mit einer zweiten, die im Bruchsack rechts in der Femoralgegend adhärent ist. Resektion des eingcklemmten Darmstückes. Enteroanastomose. Verschluß der Bruchpforte. Geheilt entlassen.

Fall 15. H. M., 49jähriger Bauer, leidend aussehender Mann. Erkrankte plötzlich an Erbrechen und heftigen Leibschmerzen, be- 
sonders in der rechten Unterbauchgegend; scit derselben Zeit vö!lige Stuhl- und Windverhaltung.

Untersuchungsbefund: Leib stark aufgetrieben, Darmversteifung sehr deutlich, keine Resistenz, Druckempfindlichkeit mäßig. Rektal o. B. Puls beschleunigt, Temperatur 38,2.

Operation: Von einer Dündarmschlinge geht ein feiner, derber Narbenstrang aus und verschwindet in einer Tasche, welche durch die Gekrösewurzel gebildet wird. Nach Lösung dieses Stranges fült sich der leere Dünndarm. In der Umgebung dieser Schlinge finden sich auch noch andere zarte Adhäsionen unter den benachbarten Schlingen. Die Tasche an der Mesenterialwurzel wird geschlossen. Punktion des Darmes. Geheilt entlassen.

Fall I6. Z. B., 65jähriger Bauer. Patient recht elend, wird im Stadium des Miserere eingeliefert.

Operationsbefund: Darmschlingen etwas gebläht, ganz träge Peristaltik. Der untere Teil des Ileums ist in einer Ausdehnung von $25-30 \mathrm{~cm}$ gangränös, cbenso die zugchörigen Teile des Mesenteriums. Die Ursache bildet die Thrombose der versorgenden Mesenterialarterie.

Operation: Resektion des crkrankten Stückes und Anastomose zwischen Ilcum und Qucrcolon. Exitus letalis.

Fall 17. G. W., mittelkräftiger Mann. An amnese: Seit einigen Tagen kein Stuhlgang mehr, kein Erbrechen.

Untersuchungsbefund: Leib stark aufgetrieben, fast bretthart, keine Schmerzempfindlichkeit, Darmsteifungen, starkes Gurren. Rektal in Reichwcite des Zeigefingers eine Stenose durch eine das ganze Darmlumen ringförmig ausfüllende, weite, zottig herabhängende Geschwulst bedingt. Es handelt sich um ein Rektumcarcinom, das den Ileus bewirkt hatte.

Operation: Anlegung eines Anus coecalis in der Gegend der Flexura dextra. Punktion des Darmcs. Operation des Carrinoms wird verweigert. Mit Pelotte entlassen.

Fall I8. B. M., 59jähriger Bauer, rüstig aussehender Mann.

Untersuchungsbefund: Leib stark aufgetrieben, bretthart, druckempfindlich, Darmsteifungen sichtbar. Rektal nihil.

Operation: Es findet sich ein stark geblähtes Coecum mo bile, das sich nach der linken Seite hinübergeschlagen hatte. Dünndarm ebenfalls stark gebläht, Dickdarm nicht gebläht, Kotballen in demselben. Nach Herüberbringen des Coecums in seine richtige Lage gehen sofort Gase in den Dickdarm. Cöcum wird an die rechte laterale Bauchwand fixiert. Exitus letalis.

Fall I9. V. G., 65jähriger Arbeiter, gut aussehend, atmet schnell. A n amnese: Erbrechen, Stuhl- und Windverhaltung.

Untersuchungsbefund: Leib stark aufgetricben; Darmversteifung sehr deutlich; keine Resistenz und Druckempfindlichkeit, 
keine Dämpfung. Rektal nihil. Keine Temperatur; Puls: Schnell, klein.

Operation: Dünndarm äußerst gebläht, lleumende kollabiert. Etwa $20 \mathrm{~cm}$ oralwärts vom Cöcum alte Adhäsionen, welche zum Teil zur Linea inominata ziehen und nicht nur das Ileum auf eine Strecke von $10-15 \mathrm{~cm}$ verengen, sondern auch die Fußpunkte dieser Schlinge nahe gebracht haben, so daß sich der oralwärts liegende Dünndarm nach aufwärts geknickt hat und so den Darmverschluß bedingt. Es wird eine Ileocolostomie gemacht. Der Dünndarm zum ' $\Gamma$ eil sehr schlecht, cyanotisch, paralytisch gebläht. Punktion desselben. Exitus letalis.

F a 1120 R. II., 24jährige, gut genährte junge Frau. A nam. nese: Krampfartige Schmerzen im ganzen Leib; Stuhl- und Wind verhaltung, Koterbrechen.

Untersuchungsbefund: Abdomen aufgetrieben, diffus druckempfindlich; während der Kolikanfälle Darmsteifung sichtba: licklal nihil.

Operation: Darmschlingen blaurot, stark aufgetrieben, Cöcum mit Ileumschlinge verklebt. Vom Cöcum zur Beckenwand (Gegend des Poupartschen Bandes) zicht eine Bride, darunter eingeklemmte Dünndarmschlinge. Bride wird abgetragen. Geheilt entlassen.

Fall 21. Ph. C., 5ojährige große, hagere Frau. An amnese: Leibschmerzen, Erbrechen, starkes Aufstoßen.

Untersuchungsbefund: Abdomen nicht aufgctrieben, weich; in der lleocökalgegend fühlt man einen geblähten, etwas druckempfindlichen Darmkanal.

Operation: Reichlich Exsudat in der Bauchhöhle, einige mäßig geblähte und verklebtc Dünndarmschlingen in der Ileocökalgegend. Bride vom kleinen Becken zum Cöcum; darunter waren einige Dünndarmschlingen durchgeschlüpft und eingeklemmt. Darm ohne Schnürfurche. Bride wird abgetragen. Geheilt cntlassen.

Fall 22. J. M., 4I jährige Bauersfrau, mittelgroß, mager, von frischem Aussehen; kolikartige Schmerzen im Leib, Kotbrcchen.

Untersuchungsbefund: Leib stark aufgetrieben, von Zeit zu Zeit sieht man durch die Bauchdecken hindurch kräftige peristaltische Bewegungen der Darmschlingen; keine Druckempfindlichkeit. Dic rektale Untersuchung ergibt eine starke Retroflexio uteri. Temperatur und Puls normal.

Operation: Dünndarm sehr stark aufgetricben, der unterste Abschnitt der Ileumschlinge kollabiert. Eine starke Bride verbindet eine Ileumschlinge mit der hinteren Wand des Uterus, fixiert die. selbe im kleinen Becken und macht sie undurchgänglich. Da die Freilegung der Schlinge mit Gefährdung der Darmwand einhergehen würde, wird eine Enteroanastomose angelegt. Geheilt entlassen.

Fall 23. M. G., Igjähriger Dienstknecht im mittleren Ernäh. 
rungszustande. An a mncse: Erbrechen und Leibschmerzen rechts unten.

Untersuchungsbefund: Geringe Spannung rechts unten, in der rechten Unterbauchgegend flache Vorwölbung, die nicht besonders druckempfindlich ist. Temperatur wenig erhöht; Puls o. B.

Operation: Aus dem Bauchideckenschnitt luxiert sich ein großes medianliegendes Gebilde, dessen Mitte und Hauptteil von dem vollkommen gangränösen Cöcum gebildet wird. Das Cöcum ist in. karzeriert und an seiner Basis läuft der ebenfalls inkarzerierte Wurm nach hinten herum. Auf seinem Wege hat er noch das Ileumende in der Länge von $10 \mathrm{~cm}$ umschnürt und ebenfalls eingeklemmt, dies in einem zweiten Halbkreis. Der Wurm läuft dann noch wieder bis vorn hin Er wira in der Mitte ungefähr durchtrennt. Dadurch wird das eingeklemmte Cöcum frei. Bei der weiteren Lösung des Wurmes wird auch das eingeklemmte Ileum frei. Der Wurm wird so vollständig abgelöst. Dann zeigen sich einige Dünndarmschlingen, die dem gangränösen Darm anlagen, nur oberflächlich schwärzlich verfärbt, sonst gut. Das prall gefüllte und ganz schwarze Cöcum mit dem lleumende (soweit dies eingeklemmt war), wird reseziert. Nahe der Flexura hepatica wird eine Ileocolostomie angelegt. Gcheilt entlassen.

Fall 24. M. D., 38jährig, verfallen aussehend. A n a mnes e: Kolikartige Schmerzen im Leib'; Stuhlgang angehalten; häufig Erbrechen und Aufstoßen; kein Kotbrechen.

Untersuchungsbefund: Abdomen aufgetrieben, prall elastisch. Von Zeit zu Zeit sieht man Darmsteifungen, die mit heftigen Schmerzen einhergehen.

Operation: Am Peritoneum zahlreiche Knötchen, ebenso am Netz. Die Darmschlingen sind zum Teil durch Adhäsionen miteinander verwachsen. Ein solcher Strang hat eine Einschnürung einer Dünndarmschlinge zur Folge und bewirkt den Strangileus. Der Strang wird gelöst und sogleich stellt sich dic peristaltische $\mathrm{Bc}$ wegungswelle wieder ein. Exsudat keines vorhanden. Es handelt sich um eine trockene Peritonitis tuberculosa mit Strangileus. Geheilt entlassen.

Fall 25. L. V., 50jähriger Mann in gutem Ernährungszustande. Anamnese: Starke Leibschmerzen mit Stuhl- und Windverhaltung. Brechreiz.

Untersuchungsbefund: Leib stark aufgetricben, Peristaltik vorhanden; Gurren zu hören.

Operation: Einige Bindegewebestränge haben den Dünndarm abgeschnürt. Nach Lösung der Stränge bläht sich der Darm schon auf. Geheilt entlassen.

F a 11 26. H. E., 56jährige Schuhmachersfrau. Bridenileus. Lösung der Bride. Geheilt entlassen. 
Fall 27. F. M., 48jährige Frau in schlechtem Ernährungszustand. A n a m nese: Seit 4 Wochen Stuhlgang selten und unregelmäßig. Appetit schlecht. Öfters starkes Leibgrimmen. Kein Abgang von Winden.

Untersuchungsbef und: Leib trommelartig aufgetrieben, deutlich fühl- und sichtbare Dünndarmsteifungen. Rektal: Reichlich Kot, nach vorn zu, nach beiden Seiten hin eine harte, ziemlich unnachgiebige Masse. Vaginal: Infiltrierte Wände, die trichterartig zusammenlaufen.

Diag nos e: Darmverschluß in Bereiche des Sigmoideum oder oberen Rectum durch lokale Metastase eines operierten Uteruscarcinoms.

Operation: Darmschlingen stark gebläht und injiziert. Anlegen eines Anus praeternaturalis. Mit Schwammbandage gebessert entlassen.

Fall 28. G. K. Invaginatio ileocolica (Mcgacolon).

F a 11 29. G., Knabe. Invaginatio ileocolica.

F all 30. Gl. M., Knabe. Invaginatio ileocoecalis.

Fall 3I. G. Postoperativer Adhäsionsileus.

Fall 32. V. M., Soldat. Invaginatio ileocoecalis.

F a 11 33. A., Anna. Steinileus.

Fall 34. B., Adolph. Volvulus.

$$
\text { Igi6. }
$$

Fa 11 35. H.W., I8jähriger, mittelkräftiger Mann. A n a mn ese: Leibschmerzen in der rechten Bauchseite.

B ef und: Leib im ganzen weich; nur Mac-Burney-Punkt druckempfindlich. Kein Fieber; kein Lrbrechen; weder Stuhl- noch Windverhaltung.

Blinddarmoperation: Nach Io Tagen Erbrechen, Stuhlund Windverhaltung. Leib trommelartig aufgetrieben.

Operation: Vom Netz hat sich ein dünner, am Becken adhärierender Strang über eine Dünndarmschlinge gelegt und diese abgeschnürt. Dünndarm stark gebläht. Netzstrang wird durchtrennt. Exitus letalis infolge Lungentuberkulose.

Fall 36. B., Adam. 5 Jahre alt, kräftig, gesund aussehend. A n a m nese: Leibschmerzen, Erbrechen, Stuhl- und Windverhaltung.

B ef und: In der Ileocökalgegend pralle, verschiebliche, kaum schmerzhafte Geschwulst.

Operation: Man findet etwa 4 bis $5 \mathrm{~cm}$ vor der Valvula Bauhini einen Schnürring am Ileum, ebenso ist dic distale Hälfte des Wurmfortsatzes leicht infarziert, desgleichen ein Appendix epiploica des Colon ascendens. Die Invagination hatte sich aber während der Narkose von selbst gelöst. Heilung.

Fall 37. B. M., I 2jährige Schreinerstochter, kräftig. Wurde 
wegen Appendicitis acuta operiert. Io Tage nach der Operation Darmsteifung, kein Stuhl, Leib aufgetrieben. Kotbrechen. Rektal nihil.

Operation: Man findet den Übergang des geblähten Darmes in den kontrahierten tief im kleinen Becken, woselbst eine Jejunumschlinge fest fixiert ist. Enteroanastomose. Geheilt entlassen.

$\mathrm{F}$ all 38. B. L., Kind von $I^{1 / 2}$ Jahren, gesund und kräftig. B e $f$ und: Leib aufgetrieben, unterhalb des rechten Rippenbogens ein ca. $8 \mathrm{~cm}$ langes walzenförmiges Gebilde zu tasten.

Operation: Das Ende des Ileum, Cöcum und fast das ganze Colon ascendens sind in das Colon transversum eingestülpt. Invaginatio ileo-colica. Desinvagination gelingt leicht. Geheilt entlassen.

F a 11 39. B. R. Ileus (Krankengeschichte fehlt).

Fall 40. H. B. Ileus (Krankengeschichte fehlt).

Fall 41. N. D. Ileus (Krankengeschichte fehlt).

F a 11 42. E. A. Ileus (Krankengeschichte fehlt).

Fall 43. St. B., 63jährige Frau in gutem Ernährungszustand. Inkompletter Ileus (Zustand nach Entfernung eines Ovarialtumors).

Operation: Anastomose zwischen Colon transversum und Flexura sigmoidea. Geheilt entlassen.

Fall 44. V. S., 56jähriger, mittelkräftiger Mann. A n a m nese: Vor 3 Monaten wegen Appendicitis acuta operiert. Jetzt heftige Schmerzen im Leib und Brechreiz; häufiges Aufstoßen.

B ef und: Leib mäßig aufgetrieben, wenig druckempfindlich.

Operation: Reichliche Verwachsungen der Darmschlingen untercinander, die schwer löslich sind. Deshalb Ileocöcostomie. Geheilt entlassen.

Fall 45. S. M., 42jährige Frau in gutem Ernährungszustand. B ef und: Leib aufgetrieben, keine pathologische Dämpfung, keine Darmsteifung.

Operation: Dünndarm unten. stark gebläht, ebenso Dickdarm bis zur Flexura lienalis. Colon transversum zum Becken heruntergeschlagen, Knickungsstelle liegt an der Flexura lienalis. An dieser Stelle finden sich alte, derbe Adhäsionen mit Darm und seitlicher Bauchwand, welche das Darmlumen einengen. Lösung der Adhäsionen. Enteroanastomose $z$ wischen Colon transversum und Flexura sigmoidea. Geheilt entlassen.

Fall 46. G. K., 3ojährige Frau in mäßigcm Ernährungszustand. An amnese: Leibschmerzen, Stuhlverhaltung, Erbrechen galliger Flüssigkeit.

B ef und: Leib sehr stark aufgetrieben und in der Mitte vorgewölbt. Überall tympanitischer Schall. Vaginal und rektal kein Befund. Hoher Puls.

Operation: Die erste Dünndarmschlinge ist in einen offenen Recessus duodeno-jejunalis hineingeschlüpft und hat nahezu den halben Dünndarm nachgezogen. Lösung des Darmes. Vernähung des Recessus. Geheilt entlassen. 
Tall 47. S. S., 71jähriger Mann in mittlerem Ernährungszustande. An amnese: Schmerzen in der linken Bauchgegend, Stuhl- und Windverhaltung, starkes Erbrechen.

B ef und: Leib aufgetrieben, gespannt, überail tympanitischer Klopfschall. Operation: Ein kleiner Zügel verbindet die untere Blinddarmschlinge mit dem Parietalpcritoneum und knickt dadurch den tiefergelegenen Blinddarm ab. Lösung des Zügels. Geheilt entlassen.

Fall 48. B. V., 68jühriger Mann in rüstigem Zustande. A n amnese: Seit I4 Tagen ganz wenig Stuhl; Winde gehen nur mäßig $\mathrm{ab}$; wenig Schmerzen.

B ef und: Abdomen stark aufgetrieben; überall tympanitischer Klopfschall. Rektal: $5-6 \mathrm{~cm}$ hoch an der vorderen Wand talergroßes, zerklüftetes, bewegliches Geschwür.

Operation: Anlegung eines Anus praeternaturalis am Colon descendens. Exitus letalis.

F a 11 49. W. F., 75 jährige, ziemlich rüstige Frau. A n a mnese: Erbrechen, wenig Abgang von Kot und Flatus.

Befund: Leib stark aufgetricben, keine sichtbare peristaltik.

Operation: Es findet sich ein das Darmlumen stenosierender Tumor der Flexura hepatica. Ileo-transverso-Anastomose. Exitus letalis.

Fall 50. B. Fr., 34jährige Frau. Chronischer Ileus durch tuberkulöses Geschwür des Colon ascendens. Exstirpation des Ulcus. Enteroanastomose. Exitus letalis.

Fall 5 I. K. M., 40jährige Frau in mittlerem Ernährungszustande. Bef und: Abdomen aufgetrieben, gespannt.

Operation: In der rechten Unterbauchgegend finden sich entzündliche Verwachsungen des Darmes mit der Bauchwand und Tube. Die Darmschlingen sind stark gebläht. die abführende Schlinge leer und kollabiert. Enteroanastomose zwischen zu- und abführendem Schenkel. Beschwerdefrei entlassen.

Fall 52. E. B., 55jährige Frau in leidlichem Ernährungszustand. Chronischer lleus durch Douglasmetastasen nach Ovarialcarcinom. Anus praeternaturalis. Exitus letalis.

Fall 53. M. A., 3jähriges Kind von mäßigem Ernährungszustand. Anamnese: Seit Geburt keinen normalen Stuhlgang; Leib war stets aufgetrieben; Stuhl immer von natürlicher Farbe. Appetit stets gut.

Befund: Der Leib stark aufgetrieben; überall Dämpfung vorhanden. Links und rechts vom Nabel sieht man den Verlauf des Colon ascendens und descendens entsprechend Vorwölbungen der Bauchdecken. Peristaltik des Darmes in mäßigem Grade zu beobachten. Rektal fühlt man an der Vorderseite des Rectum weite Schleimbautfalten. 
Operation: Nach Eröffnung des Abdomens kommt ein stark dilatierter Dickdarm zum Vorschein, der das Gebiet der Flexur und des Colon transversum einnahm. Colon ascendens war nicht aufgetrieben. Flexur und Colon transversum sehr lang, Wandung verdickt und etwas brüchig. Der Dickdarm wurde bis zur Mitte des Colon transversum losgelöst, Durchtrennung im Übergang von Flexur im Mastdarm. End-zu-Seit-Anastomose zwischen Colon transversum und Mastdarm. Durchmesser des $24 \mathrm{~cm}$ langen resezierten Darm. stückes: oben (Colon transversum) $4 \mathrm{~cm}$, an der Flexur $5 \mathrm{~cm}$, unten $9 \mathrm{~cm}$. Exitus letalis infolge starker postoperativer Blutungen im Operationsgebiet.

Fall 54. M. P., 54jähriger Mann in mittlerem Ernährungszustande. An a mne se: Starke Schmerzen im Unterleib; Erbrechen; Stuhlverhaltung.

B ef und: Abdomen etwas vorgewölbt, weich, leicht angedeutete Darmsteifungen. Im Magen dünnflüssiger Kot. Rektal nihil.

Operation: Das obere Drittel des Dünndarms ist durch eine kleine Spange abgeschnürt und aufgetrieben. Lösung der Spange und Entleerung des Darmes. Geheilt entlassen.

Fall 55. L., Mathilde. Ileus durch Rectumcarcinom (Kran. kengeschichte fehlt). fehlt).

F all 56. L., Wilburgis. Chronischer Ileus (Krankengeschichte 1917.

Fal1 57. E. J., 67jähriger Mann. B ef und: Leib aufgetrieben, bretthart gespannt, außerordentlich stark druckempfindlich; über dem ganzen Leib tympanitischer Schall; Puls kräftig, voll. Temperatur normal. Zunge stark belegt, ziemlich trocken.

Operation: Eine über $\mathrm{I} \mathrm{m}$ lange Dünndarmschlinge ist von einem Zügel abgeschnürt und vollkommen gangränös geworden. Resektion der ganzen Schlinge und Enteroanastomose. Geheilt entlassen.

F a 11 58. F., Agnes. Strangileus. Krankenblatt fehlt.

Fal1 59. R. S., 69jährige rüstige Frau in mittlerem Ernährungszustande. A n a mne s e: Öters Erbrechen, auf Klystier reichlich Stuhl, jedoch nie Flatus.

B ef und: Leib in toto aufgetrieben, mäßig druckempfindlich, keine Resistenz. Nach Palpation Peristaltik ausgelöst, Darmsteifungen in Nabelhöhe. Rektal nihil.

Operation: Stark geblähte Dünndarmschlingen, nach dem kleinen Becken zu leere Schlingen. Als Hindernis findet sich ein etwa walnußgroßer Gallenstein, der das Dünndarmlumen vollstänđig verschließt. Darm in gutem Zustande. Stein wird entfernt, Darm wieder geschlossen. Greheilt entlassen.

Fa il 6o. F., Regina. Chronischer Ileus. Krankenblatt fehlt. 
Fall 6r. B. L., 4jähriger Junge in gutem Ernährungszustande. A namnese: Starke Leibschmerzen, öfters Erbrechen, Stuhl- und Windverhaltung.

B ef und: In der rechten Leibgegend fühlt man einen harten, nach oben ziehenden Tumor, der anscheinend mit Colon ascendens identisch ist. Gegend stark druckempfindlich.

O peration: Es handelt sich um eine Invagination. Resektion des Colon ascendens und Teil des Quercolons. Seitenanastomose zwischen Endteil des Dünndarms und Quercolons. Exitus letalis.

Fall 62. L... Auguste. Gallensteinilcus. Krankenblatt fehit.

Fall 63. 5jähriger Junge von gesundem Aussehen und gutem Ernährungszustand. B c f und: Leib mäßig aufgetrieben, starke Muskelspannung.

Operation: Es findet sich etwa $30 \mathrm{~cm}$ vor der Klappe ein etwa $5 \mathrm{~cm}$ langes Meckelsches Divertikel, Dünndarm s-förmig verschlungen, zeigt cine schr starke, fast durchsichtige Schnürfurche. Dünndarm hypertrophisch, gebläht. Resektion eines $\mathrm{ca} .30 \mathrm{~cm}$ langen Darmabschnitts. Seit-an-Seit-Anastomose. Exitus letalis.

F all 64. M. B., 64jährige mittelgroße, magere Frau von schlechtem Aussehen. A n a mnese: Bauchschmerzen und Erbrechen, Stuhlund Windverhaltung. Kotbrechen.

B ef und: Abdomen stark aufgetrieben, deutliche Dünndarmsteifungen, kein Tumor palpabel. Rektal Druckempfindlichkeit des hinteren Douglas.

Operation: Es finden sich stark geblähte Dünndarmschlingen, Dickdarm lcer. Im kleinen Becken ist cine Dünndarmschlinge dem Fundus uteri adhärent. Zwischen dieser und dem Cöcum ist eine weitere Schlinge durchgeschlipft und abgeknickt. Beim Lösen vom Uterus reißt der Darm ein, außerdem ist an der Abknickungsstelle der Schnürring stark verdünnt. Resektion von ca. $15 \mathrm{~cm}$ Dünndarm. Seit-an-Scit-Anastomose. Exitus letalis.

Fall 65. K. Fr., 23jähriger Mann, macht einen kranken Eindruck. B efund: Leib mäßig aufgetrieben, überall starke Muskelspannung, überall Schmerzempfindlichkeit.

Operation: Es wird ein Meckelsches Divertikel vorgefunden, das über eine Dünndarmschlinge hinübergelagert und am Mesenterium ciner anderen Schlinge adhärent ist. Die ganze Partie ist mit Fibrin bèlegt. I) Schnürfurchen der Dünndarmschlingen erweisen sich die eine als gut, die andere sehr dünn, am Durchbruch. Dünndarm zeigt keine Peristaltik. Resektion des Divertikcls und Dünndarmanastomose, Seit-an-Seit. Exitus letalis.

Fall 66. Kr. A., I gjähriger, etwas blasser, wenig kräftiger Mann. An a mnese: Starke Leibschmerzen, Erbrechen; viel Auf stoßen, Stuhl- und Windverhaltung.

B ef und: Leib aufgetricben, Muskelspannungen, stärkerer Druckschmerz im Epigastrium. Puls beschleunigt, Temperatur normal. 
Operation: Dünndarmschlingen hochgradig gebläht und gerötet zeigen nur geringe Bewegung. Die unterste Dünndarmschlinge etwa $10 \mathrm{~cm}$ vom Cöcum entfernt durch Narbenstrang quer vollkommen abgeschnürt. Der Strang reißt bei Manipulation mit dem Darm, da jedoch die Schnürfurche völlig nekrotisch erscheint, wird Resektion des Dünndarmabschnitts vorgenommen. Enteroanastomose Seit-an-Seit. Geheilt entlassen.

F all 67. Kr., Georg, Soldat. Ileus bei Peritonitis tuberculosa. Krankengeschichte fehlt.

F a 11 68. S., Anna. Ileus (Strang) bei Peritonitis tuberculosa. Krankengeschichte fehlt.

Fall 69. Sch., Josef, Soldat. Ileus bei Peritonitis tuberculosa. Krankengeschichte fehilt.

F all 70. B. H., 37jährige Frau in mittlerem Emährungszustand. Anamese: Heftiges Erbrechen, Stuhl- und Windvcrhaltung.

B ef und: Abdomen stark meteoristisch aufgetrieben, mit deutlichen Darmsteifungen. Rektal dicht hinter der Portio mit dieser verwachsen, ein derber, wenig druckempfindlicher, knotenförmiger Tumor. Carcinoma recti.

Operation: Darm stark gebläht; ein Anus practernaturalis angelegt. Wird mit Bandage entlassen.

F all 7I. G. B., 27jährige Frau in mittlerem Ernährungszustand. Tumor der Valvula Bauhini, der das Darmlumen vollständig verschliebt. Ilcocolostomie. Geheilt entlassen.

Fall 72. St. W., 29jährige Frau in gutem Ernährungszustand. A n a m n e se: Erkrankt an heftigen kolikartigen Schmerzen im Leib nach reichlichem Genuß von Kirschen; starkes Erbrechen; Verhaltung von Stuhl und Winden. Temperatur etwas erhöht, Puls 104.

B ef und: Abdomen in toto stark aufgetrieben und gespannt, überall druckempfindlich, nirgends Darmsteifungen, nirgends ein Tumor rektal und vaginal.

Operation: Dünndarm in toto stark gebläht ohne Peristaltik, Dickdarm leer. An der Valvula Bauhini findet sich das Cöcum fest nach unten fixiert, das stark geblähte und gefüllte Ileum führt in einem starken Abknickungswinkel von oben her darauf zu. Im Ileum sind zahlreiche Kirschkerne zu fühlen. Es ist also durch das Gewicht der Stcine und durch starke Füllung des Dünndarms eine Abknickung zustande gekommen. Ileocolostomie. Exitus letalis.

F a 11 73. B. E., 68jährige Bauersfrau. Ileus infolge eingeklemmter rechtsseitiger Schenkelhernie. Moribund eingeliefert. Exitus letalis.

F all 74. E., Georg, 49jähriger Mann. An a mnese: Kotiges Erbrechen, Stuhl- und Windverhaltung. lichkeit.

Befund: Leib stark aufgetrieben, überall Druckempfind- 
Operation: Uberall ist der Darm mit der Haut verwachsen und stark gebläht. Enterostomie. Exitus letalis.

Fall 75. K. G., 44jähriger Mann in gutem Ernährungszustand. Bei der Operation findet man den größten Teil der Blinddarmschlingen gebläht vor. Fine solche Schlinge ist rechts an der vorderen Bauchwand gegen den Darmbeinkamm zu adhärent und abgeknickt. Lösung der Dünndarmschlinge. Enteroanastomose. Geheilt entlassen.

Fall 76. K. H., I 5jähriges Mädchen in gutem Ernährungszustande. Bei der Operation findet sich das Colon descendens und sigmoid. einmal torquiert und zur rechten Bauchseite hingeschlagen. Geheilt entlassen.

F a 11 77. J. E., 32jährige Frau in mäßigem Ernährungszustand. DarmverschluB durch Carcinom der Flexura linealis. Geheilt entlassen.

Fall 78. M., Magd., 43jährige Frau in äußerst schlechtem Zustand. Bei der Operation finden sich zwei Stränge, die von den Genitalien nach oben ziehen und die Darmstenose bedingen. Durchtrennung der Stränge. Exitus letalis.

Fall 79. G., Math., 47jährige Bauersfrau. Bei der Operation findet sich eine Dünndarmschlinge von einem kurzen Strang fixiert und geknickt. Durchtrennung des Stranges. Heilung.

Fall 8o. B. A., 77jähriger Mann in sehr elendem Zustand. Bei der Operation findet sich eine stark gefüllte Blase, welche den Darm komprimiert hat. Prostatahypertrophie. Exitus letalis.

F all 8I. L., Anna. Ileus bei Hernia femoralis incarcerata. Kंrankenblätter fehlen.

F a 11 82. Sch., Willy. Ileus bei Torsion des Sigmoideum. Krankenblatt fehit.

Fall 83. K., Hermann. Ileus. Krankenblatt fehlt.

F a 11 84. R., Stefan. Ileus. Krankenblatt fehlt.

\section{8.}

Fa 1185. Kn., Eva. Adhäsionsileus. Krankenblatt fehlt.

Fal1 86. M., J., 50jährige Frau in mittlerem Ernährungszustand. Bei der Operation findet sich der Darm der Bauchwand breit adhärent. Der Dünndarm ist hypertrophisch, stark gefüllt, der abführende Teil ist leer. Der Darm findet sich an der Einklemmungsstelle einer Hernia fem. sin. verbacken und dadurch abgeknickt. Resektion der verengten Partie und Seit-an-Seit-Anastomose. Heilung.

F a 11 87. St., Ott., 22jähriges Dienstmädchen in mittlerem Ernährungszustand. Vom Cöcum zum Mesenterium zicht ein bleistiftdicker Strang, unter den der Dünndarm durchgeschlüpft,ist. Durchtrennung des Stranges. Heilung. 
F all 88. N. M., 55jährige Frau, blaß, abgemagert. Die Operation ergibt eine Invagination des Dünndarms in Ausdehnung von $40 \mathrm{~cm}$. Bei Repositionsversuchen reißt die Serosa an einer Stelle ein. Von Reposition wird daher Abstand genommen und das ganze Darmstück etwa $120-130 \mathrm{~cm}$ reseziert. Seit-an-Seit-Anastomose. Heilung.

F all 89. 6ojährige, elend ausschende magere Frau. O pera tion: Stark geblähter Dünndarm, in der rechten Unterleibsseite leerer Darm. Hier findet sich eine frisch entzündliche Adhäsion und der Darm ist abgeknickt. Exitus letalis.

Fall go. H. E., 6jähriges Kind in schwerkrankem Zustand. Operation: Der Dickdarm ist mit der vorderen Bauchwand verklebt, wodurch ein Passagehindernis zustande kam. Heilung.

F all 91. Sch., B., 67jährige kräftig gebaute Frau. Ileus infolge stenosierendem Rectumcarcinoms. Exitus letalis.

Fall 92. B., M., 69jährige hagere Frau in gutem Allgemeinzustand. lleus des Dickdarms, bedingt durch ein stielgedrehtes, rechtsseitiges Ovarialkystom von etwa Mannsfaustgröße und ein etwas kleineres, linksseitiges Kystom. Heilung.

Fall 93. A., Martha, 68jährige Frau in gutem Ernährungszustand. Ileus infolge stenosierendem Carcinoma uteri. Anus praeternaturalis. Mit Bandage entlassen.

Fall 94. L. G., 74jähriger Mann in guter körperlicher Verfassung. Operation: Es findet sich eine Stelle, wo eine Dünndarmschlinge mit dem Mesenterialansatz der gegenüberliegenden Seite mit einer höher gelegenen Schlinge und an deren Mesenterialansatz verwachsen ist. An der Stelle der Verwachsung besteht eine tiefe Einschnürung. Lösung der verwachsenen Schlingen. Exitus letalis.

Fall 95. U. S., 52jährige Frau von blasser Gesichtsfarbe, mäßigem Fettpolster. Carcinoma uteri et recti, Ascites mit Ileuserscheinungen. Exitus letalis.

Fall 96. Sch., W., 6rjähriger Mann. Chronischer DarmverschluB infolge stenosierenden Carcinoms der Flexura linealis des Dickdarms. Exitus letalis.

F all 97. F. R., 3jähriges, schwächliches, mäßig entwickeltes Kind. Operation: Es zeigt sich, daß am Cöcum auf der Außenseite sich die Darmwand fingerhutartig eingestülpt hat. Die Wandung ist an dieser Stelle deutlich verdickt und etwas entzündet. Da ständig die Gefahr zu vollkommener Invagination besteht, wird das unterste Ileum mit dem Cöcum reseziert. Ileotransversostomie. Seitliche Enteroanastomose. SchluB des Schlitzes im Gekröse. Heilung.

Fall 98. B., Marzella, 48jährige, kräftig gebaute Frau. Operation: Es zeigt sich, daß Dünndarmschlingen an mehreren Stellen stark verwachsen und stark abgeknickt sind. An einer Stelle- 
ist der Dünndarm bis Bleistiftdicke verengt. Antiperistaltische Enteroanastomose zwischen geblähtem und kollabiertem Darm. Exitus letalis.

F a 11 99. B., Chr., 41jährige, elend und verfallen aussehende Frau in mittlerem Ernährungszustand. Operation: Der Dünndarm ist in toto stark gebläht und gefüllt. Als Ursache der Stenose findet sich ein stricknadeldünner derber Strang, der vom kleinen Becken zum Cöcum führt und unter dem Darm durchgeschlüpft ist. Durchtrennung des Stranges. Der Einschnürungsring wird übernäht. Enteroanastomose. Seit-zu-Seit. Exitus letalis.

F a 11 ıoo. G. G., 7Ijähriger, für sein Alter rüstiger Mann von gutem Aussehen. Operation: Es liegen geblähte und injizierte Dünndarmschlingen vor, die untersten Dünndarmabschnitte sind leer. Am Übergang des geblähten zum leeren Darm findet sich ein deutlicher Schnürring eines Darmwandbruches, der sich spontan gelöst hat. Die eingeklemmte Darmwand ist relativ gut, so da $B$ sich eine Resektion oder Übernähung erübrigt. Exitus letalis.

Fall Ior. K., E., I7jähriges graziles Mädchen in mittlerem Ernährungszustand. Operation: Es findet sich das lleum in den untersten Partien in Ausdehnung von $30 \mathrm{~cm}$ zu einem dichten Konvolut von Schlingen verbacken und von einem Schleier von Fibrin belägen überzogen. Resektion des Dünndarms und Ileocoiostomie. Heilung.

Fall 102. H. J., 68jähriger Mann, rüstig. Befund: Epigastrium etwas eingewachsen. In der rechten Bauchgegend fühlt man einen prallen, glatten, walzenförmigen Tumor, der Verschieblichkeit zeigt. Keine Darmsteifungen. Über dem Tumor gedämpfter Schall. Operation: Eine Invagination des Cöcum in das Colon transversum wird gefunden. Vom Cöcum ist nur noch der Wurm zu sehen. Da Lösung der Invagination nicht gelingt, wird das ganze Darmstück reseziert. Blinder Verschluß des Transversum und Ileum. Ileocolostomie. Seit-an-Seit. Heilung.

Fall 103. B., K., 55jährige Frau, elender Allgemeinzustand. Operation: In der Ileocökalgegend und weiter nach oben ein Knäuel verwachsener und verschlungener Dünndarmschlingen. Beim Versuch der Lösung entleert sich aus der 'Tiefe sofort Darminhalt. Die Därme reißen dauernd ein, ein $30 \mathrm{~cm}$ langes Stück, das vollständig gangränös ist, wird entfernt und eine Seit-an-Seit-Anastomose ausgeführt. Auf eigenen Wunsch in moribundem Zustande nach Hause entlassen.

Fall 104. R., E., 62jährige Frau, wurde moribund mit Ileuserscheinungen eingeliefert. Dic Obduktion ergab Einklemmung des untersten Ileums durch einen bindegewebigen Strang des großen Netzes. Der Strang inseriert ungefähr $3 \mathrm{~cm}$ neben der rechten Flexur am Colon transversum. 
F all 105. W., E., 5ojährige Frau, sieht sehr angegriffen aus. Operation: Dünndarm gefüllt mit flüssigem Darminhalt, an ein. zelnen Stellen Verwachsungen. Colon kollabiert. Der Úbergang des Ileums ins Colon ist durch einen ziemlich dicken Strang, der vom Uterus ausgeht, komprimiert, nach dessen Durchtrennung die Passage frei wird. Exitus letalis.

F all ı6. B., B., 6ijährige Frau. Ileus infolge Carcinoms der Gallenwege mit Carcinose des Peritoneums. Exitus letalis.

Fal1 107. Sch., A., I8jähriges Mädchen in leidlichem Ernährungszustand. Operation: In der Nabel- und rechten Oberbauchgegend sind Darmschlingen, Netz und Peritoneum ziemlich verklebt. In der linken Oberbauchgegend liegt ein ganzes Bukett von Darmschlingen, die durch eine Bride abgeschnürt sind. Die Darmschlingen sind in diesem Konvolut fast alle miteinander ziemlich verklebt. Lösung der Verwachsungen, Abtragung und Unterbindung der Bride. Heilung.

F all 108. E., Maria, 3ojährig. Wurde mit Ileuserscheinungen eingeliefert; $1 / 4$ Stunde nach der Einlieferung trat der Tod ein. Obduktion ergab stark geblähte Darmschlingen. Diplokokken. Peritonitis. 21 Eiter werden aus der Bauchhöhle und dem kleinen Becken entfernt.

F all 109. N. J., 67jähriger Mann. Adhäsionsileus. Entero stomie. Exitus letalis.

Fall I Io. B., R., 74jährige Frau in dürftigem Ernährungszustand. Operation: Ungewöhnlich großes Ovarialkystom, das das obere Jejunum komprimiert hat. Exitus letalis.

Fall 111. B., Hans. Ileus infolge Carcinoma recti. Krankenblatt fehlt.

F a 11 I 2. Z., Adam. Strangileus. Krankenblatt fehlt.

Fall Ir3. G., Marg. Ileus. Krankenblatt fehlt.

\begin{tabular}{|c|c|c|c|c|c|c|c|}
\hline Nr. & \multicolumn{2}{|c|}{ Ileus-Arten } & 1914 & 1915 & 1916 & 1917 & 1918 \\
\hline I & Dynamischer Ileus & $\begin{array}{l}\text { 1. Paralytischer Ileus } \\
\text { 2. Spastischer Ileus }\end{array}$ & - & 1 & $\underline{I}$ & - & - \\
\hline 2 & Mechanischer Ileus & $\begin{array}{l}\text { I. Okklusions-Ileus } \\
\text { 2. Obturations-Ileus } \\
\text { 3. Kompressions-Ileus } \\
\text { 4. Stenosen-Ileus } \\
\text { 5. Inflektions-Ileus } \\
\text { 6. Strangulations-Ileus }\end{array}$ & $\begin{array}{l}\overline{2} \\
- \\
\overline{7} \\
\mathrm{I}\end{array}$ & \begin{tabular}{|}
5 \\
3 \\
9 \\
5
\end{tabular} & $\begin{array}{l}\overline{1} \\
6 \\
8 \\
2\end{array}$ & $\begin{array}{c} \\
3 \\
1 \\
3 \\
11 \\
5\end{array}$ & $\begin{array}{l}\overline{3} \\
3 \\
5 \\
16 \\
1\end{array}$ \\
\hline 3 & $\begin{array}{r}\text { Ileus-Fälle, bei der } \\
\text { blätter die Art d } \\
\text { wer }\end{array}$ & $\begin{array}{l}\text { en mangels der Kranken- } \\
\text { es IJeus nicht festgestellt } \\
\text { den konnte }\end{array}$ & - & - & 5 & 5 & $I$ \\
\hline & & & 10 & 23 & 23 & 28 & 29 \\
\hline
\end{tabular}


Nebenstehende tabellarische Übersicht möge zunächst einen klaren Überblick verschaffen! Wenn wir näher darauf eingehen, so sehen wir, daß der dynamische Ileus (paralytischer 2 Fälle, spastischer Ileus o) in verschwindender Minderzahl ist. Bei den beiden Fällen des paralytischen Ileus handelt es sich bei dem einen (Fall I6) um eine Thrombose der versorgenden Mesenterialarterie und infolgedessen Gangrän des untersten Teiles des Ileums in einer Ausdehnung von $20-25 \mathrm{~cm}$; bei dem zweiten (Fall 43) um eine nach einem operativen Eingriff (postoperativer lleus) entstandene Störung der Darmpassage. Beim mechanischen lleus steht bezüglich der Häufigkeit an erster Stelle der Inflektionsileus, der $27 \mathrm{mal}$ hervorgerufen wurde durch Abschnürung in-folge eines Stranges, 2omal durch Adhäsionen, 2mal fand sich ein Meckelsches Divertikel, 2mal Hirschsprungsche Krankheit und Imal Treitzsche Hernic (zusammen 52 Fälle). Dann folgt bereits in großem Abstand (17 Fälle) der Stenosenileus, hauptsächlich bedingt durch Carcinom des Darmes, vor allem des Rektums. Daran schließt sich mit 14 Fällen der Obturationsileus, unter denen sich Io Invaginationen, zwei Verschlüsse durch Gallensteine, je einer durch Kotmassen und Stein finden. An 4. Stelle kommt der Strangulationsileus mit 6 Volvulus, 5 Inkarzerationen, 2 Torsionen, zusammen 13 Fällen. Der Kompressionsileus ist nur mit 4 Fällen vertreten und wurde bedingt 2mal durch Ovarialkystome, I mal durch prolabierten Uterus, I mal durch dic stark gefüllte Blase bei Prostatahypertrophie. Von I I Fällen konnte leider die Art des Darmverschlusses nicht festgestellt werden, da die nötigen Unterlagen nicht aufgefunden werden konnten.

Wenn wir einen kurzen Blick auf das Alter der Patienten werfen, so finden wir, daß die meisten Erkrankungen zwischen dem 30. und 6o. Lebensjahre, gleichviel von $\mathrm{I}-30$ und über 6o Jahre stattfanden und ungefähr die doppelte Anzahl weibliche Patienten sich finden als männliche. Weitaus das Hauptkontingent (9o Proz.) stellt das Land. Geheilt oder in Besserung wurden ungefähr 60 Proz. entlassen, während ungefähr 40 Proz. mit Exitus letalis abgingen.

Die tabellarische Übersicht lehrt uns, daß die Erkrankungen von Jahr zu Jahr an Häufigkeit zunahmen. Noch deutlicher wird 
dies, wenn wir die beiden Jahre vor dem Kriege zum Vergleiche heranziehen. Es fanden sich in Jalıre:

\begin{tabular}{|c|c|c|c|c|}
\hline 1912 & 5 & äll & vor & Ileus \\
\hline 1913 & 10 & $"$ & , & \\
\hline 1914 & IO & $"$ & ", & \\
\hline 1915 & 23 & , & ", & \\
\hline 1916 & 23 & ," & ", & \\
\hline 1917 & 28 & " & , & \\
\hline 1918 & 29 & ״ & $"$ & \\
\hline
\end{tabular}

Im einzelnen ist nun vor allem die Steigerung des Inflektions(bes. Strang- und Adhäsions)Ileus, des Strangulationsileus (vor allem der Inkarzeration) und des Volvulus (19r8 zeigt sich freilich hier ein Rückgang) wahrzunehmen.

Daß diese auffallende Vermehrung des Darmverschlusses, die auch in anderen Kliniken beobachtet wurde - so berichtet $\mathrm{K} \ddot{\mathrm{n}} \mathrm{ig}$ aus der Marburger Klinik, D o os e aus dem allgemeinen Krankenhaus Lübeck über auffallende Zunahme des mechanischen Ileus - auf die durch den Krieg veränderte Lebensweise zurückzuführen ist, darf wohl mit aller Sicherheit angenommen werden. Vor allen Dingen ist wohl der Kriegskost die Hauptschuld an der starken Zunahme der Darmpassagestörungen in die Schuhe zu schieben. Daß die Kriegskost einen enormen Einfluß auf den Frnährungszustand des Menschen - konnte man doch, wenn man nach längerer Abwesenheit im Felde auf Urlaub nach Hause kam, einen früher wohlbeleibten Bekannten schlank und an Körpergewicht bedeutend zurückgegangen wiederfinden - und die Tätigkeit des Darmtraktus ausübt, steht außer jedem Zweifel.

Eine kurze Betrachtung über die Zusammensetzung der Kriegskost wird zur Klärung dieser Tatsache beitragen. $Z$ untz schildert in einer Arbeit - Einfluß des Krieges auf Ernährung und Gesundheit des deutschen Volkes. Med. Klinik I9I5 - die Hauptcharakteristica der Kriegsnahrung folgendermaßen:

„Die wesentlichen Änderungen sind Einschränkung des Fleischgenusses, reichlichere Benutzung von Kartoffeln und Verwendung kleiehaltigen Brotes. Namentlich das letztere kann leicht Verdauungsstörungen hervorrufen. Die mechanischen Leistungen der Darmmuskulatur erleiden mit jeder Änderung der physikalischen und chemischen Eigenschaften des Darminhalts Veränderungen, durch welche sie sich den neuen Verhältnissen anpassen. Diese Veränderungen 
sind bei plötzlichem Wechsel der Lebensweise sehr stark, sie gehen in negativem oder positivem Sinne über das Wünschenswerte hinaus. Wenn die neue Nahrung erhöhte mechanische Arbeit fordert, kommt es zu abnormer Peristaltik, die schlechtere Ausnützung der Nahrung. Durchfalle und die bekannten damit verbundenen Beschwerden auslöst. Ist die neue Nahrung dagegen eine reizlosere als gewöhnlich, so tritt Verstopfung und die aus ihr resultierenden Beschwerden auf, bis sich das Darmnervensystem an die neuen Verhältnisse angepaßt hat. Die normale Gärung im Blinddarm und Dickdarm bewirkt bekanntlich in erster Linie eine ausgiebige Lösung der Zellulose, die unter Bildung von flüchtigen Fettsäuren, von Methan und Kohlensäure zerfällt, worauf die entstandenen Fettsäuren als Nährstoffe benutzt werden. Wenn wir einen Menschen, der früher nur wenig Zellulose mit seiner Nahrung aufnahm, reichlich grüne Gemüse oder kleiehaltiges Brot geben, so bedingt die dadurch leicht stärker werdende Dickdarmgärung leicht Beschwerden. Die flüchtigen Fettsäuren sind ein starker Reiz für die Darmbewegung, der zu Durchfall und im AnschluB daran zu schlechter Ausnützung der Nahrung führt."

Neben der starken Vermehrung des Zellulosegehaltes der Kriegskost, die eine vermehrte Peristaltik bedingt, ist noch vor allen Dingen der geringe Gehalt der Kriegsnahrung an Fett von Wichtigkeit.

Diese fettarmen, zellulosereichen Nahrungsmittel bedingen einen starken Fettschwund und erhöhen bedeutend die Darmtätigkeit. Die durch die mangelhafte Fettzufuhr bedingte Abmagerung erstreckt sich nicht nur auf das subkutane Fettpolster, sondern auch auf das subseröse Fett des Bauchfells. Dieser Schwund des mesenterialen Fettes läßt das vorher straffe Mesenterium schlaff und nachgiebig werden und verlängert dasselbe.

Dadurch wird der Darm bedeutend beweglicher. Sind nun die Bedingungen zum inneren VerschluB, sei es in Form von Adhäsionen oder eines Stranges, eines Meckelschen Divertikels usw. gegeben, so kann man sich leicht vorstellen, daB der abnorm gefüllte und geblähte Darm mit seiner erhöhten Tätigkeit und abnorm großen Beweglichkeit z. B. unter einem Strang hindurchschlüpft oder infolge von Verwachsungen abknickt. Es besteht also kein $Z$ wcifel, daß3 die durch die Kriegskost herbeigeführten Verhältnisse prädisponierend sind für eine mechanische Komplikation des Magen. Darmkanals. 
Bei Inkarzeration und Volvulus ist noch viel leichter ein stichhaltiger Beweis für den Einfluß der Kriegskost auf die Vermehrung des Darmverschlusses zu finden. Während bei normaler Nahrungszufuhr die bestehenden Bauchfelltaschen infolge des reichlich vorhandenen Fettes nur angedeutet sind, werden sie durch den Schwund des subserösen Fettgewebes mehr oder weniger klaffend. Der nun infolge des fettarmen, verlängerten Mesenteriums und durch die erhöhte Peristaltik der Zellulosenahrung abnorm bewegliche Darm findet mit viel mehr Sicherheit eine Gelegenheit, durch die Bauchfelltasche durchzuschlüpfen und abzuknicken. Eine günstige Vorbedingung für das Zustandekommen des Volvulus bildet ein langes, schmales Mesenterium, da die Fußpunkte des Darmes einander genähert werden. Die Verschmälerung des Mesenteriums wird teils auf angeborene Anlage, teils auf Schrumpfungsvorgänge und Narbenbildung zurückgeführt. Als weiteres Moment ist jedoch der Schwund des mesenterialen Fettgewebes anzuführen. So erwähnt $K o ̈ n i g$, daß cin langes fettarmes Mesenterium in einer weiten Bauchhöhle günstig für das Zustandekommen eines Volvulus ist. Nothnagel nimmt als Vorbedingung für die Entstehung von Achsendrehungen und Verknotungen des Darmes mit an erster Stelle: Magerkeit der Bauchdecken, Fettmangel im Omentum und Mesenterium. Auch Wilms sieht die infolge allgemeiner Abmagerung fortschreitende Schrumpfung des Mesenteriums als günstiges Moment für das Zustandekommen, des Volvulus an. Wenn wir nun noch bedenken, daß infolge des Nahrungsmittelmangels während der Kriegszeit ein größeres Intervall zwischen den einzelnen Mahlzeiten eintrat als vorher, so stellt sich der Vorgang beim Zustandekommen des Volvulus wie folgt dar.

Der infolge einer längeren Hungerperiode leere Darm wird mit großen schwerverdaulichen, zellulosereichen Nahrungsmitteln gefüllt. Der an einem infolge der bisherigen Zellulosekost langen, fettarmen Mesenterium hängende und abnorm bewegliche Darm, der überdies durch die Menge der aufgenommenen Nahrung sehr schwer und in starker peristaltischer Bewegung ist, sinkt nach unten und der Volvulus ist zustande gekommen. Als begünstigendes Moment kommt noch hinzu, daß die unteren Darmabschnitte leer sind und deshalb keinen Widerstand entgegensetzen 
und daB die Fußpunkte des Darmes infolge des langen schmalen Mesenteriums einander genähert sind.

Im übrigen ist eine längst bekannte Tatsache, daß in Ländern, in denen auch in Friedenszeiten eine unserer Kriegskost ähnliche fettarme und zellulosereiche Nahrung genossen wird, Passagestörungen des Darmes viel häufiger vorkommen als bei uns. So berichtet Spasokukozky aus der chirurgischen Abteilung des Gouvernements-Krankenhauses in Smolensk das häufige Auftreten von Darmverschluß, hauptsächlich durch Volvulus bedingt, bei den Russen. Diese Tatsache wird auf die relativ größere Länge des russischen Darmes (Russen 26, Deutsche I $6 \mathrm{FuB}$ ), bedingt durch die fast ausschließlich vegetarische Kost der Russen, besonders der Landbevölkerung, zurückgeführt. Luksch weist auf der Naturforscherversammlung Meran 1915 (Pathologischer Kongreß) auf das häufige Vorkommen des Darmverschlusses (die Hälfte der chirurgischen Patienten leide an Darmverschluß) bei den Bauern in der Bukowina hin und nimmt als Ursache die vorwiegend vegetabilische Kost der Landbevölkerung an. F alt in führt den in Finnland so häufig vorkommenden Cöcumvolvulus auf die in der Hauptsache aus saurem Brot und Kartoffeln bestehende Nahrung der finnischen Bauern zurück. Auch bei unseren Fällen stammen weit die Mehrzahl der Patienten (9o Proz.) vom Lande. Nicht unerwähnt will ich eine Mitteilung von Brunzel (Deutsche Zeitschrift für Chirurgie, Bd. 145) lassen, der über eine eigenartige Form des paralytischen Ileus nach Genuß roher Vegetabilien, beobachtet auf der chirurgischen Abteilung des Krankenhauses Braunschweig, berichtet.

„Nach reichlichem Genuß roher Vegetabilien kann infolge rapid einsetzender Gärungsvorgänge im Darmkanal das Bild eines Ileus mit oft ganz enormer Auftreibung des Leibes entstehen."

Ferner möchte ich nicht vergessen $z u$ erwähnen, daß als letztes auslösendes Moment des Darmverschlusses die vermehrte schwere Arbeit, die der einzelne während des Krieges infolge des Mangels an Arbeitskräften zu leisten hatte, und der Umstand, daß viel Leute, die vorher die Arbeit nicht gewohnt waren, schwere Arbeit auf sich nehmen mußten, in Betracht kommt.

Es sei noch gestattet, einige Worte über die Symptome des Darmverschlusses zu sagen. Mitten im besten Wohlbefinden über- 
raschen den Kranken heftige Kolikschmerzen und bald tritt Erbrechen ein. Dazu gesellen sich bald völlige Verhaltung des Stuhles und der Winde. Der Leib wird aufgetrieben und zeigt meist starke Druckempfindlichkeit. Dazu kommt noch Aufstoßen. Allmählich werden galliggrüne, dünnflüssige, bittere Massen erbrochen, die bald fäkulant riechen, und schließlich wird Kot erbrochen. Die Extremitäten werden kühl, Nase und Ohren livide. Schließlich geht der Kranke an Erschöpfung zugrunde, wenn nicht schon durch Komplikationen (Peritonitis, Aspirationspneumonie infolge des Erbrechens) der Tod eingetreten ist.

Dic Prognose ist immer ernst und der Darmverschluß führt immer in kürzerer oder längerer Zeit zum sicheren Tode, wenn nicht durch einen operativen Eingriff das Passagehindernis beseitigt wird. Ganz wenige Fälle sind bekannt, wo sich das Pas. sagehindernis gelöst hat und der Darm wieder durchgängig wurde. Lis ist daher die Pflicht des zugezogenen Arztes, auch wenn nur der Verdacht auf Darmverlegung besteht, in kürzester 7eit dafür zu sorgen, daß durch einen operativen Eingriff Hilfe gebracht werden kann.

Zum Schluß sei noch erwähnt, daß auch im Jahre 1919es wurden bis zum 15. II. bereits 4 Fälle operiert - die Häı. fung des lleus anhält. Wic aus vorstehenden Erörterungen her. vorgeht, wird auch wohl kaum eine Änderung zu erwarten sein, bevor sich die Ernährung wieder auf eine solche, wie sie in Friedenszeiten bestand, eingestellt hat.

Es ist mir noch ein Bedürfnis, Herrn Geh. Hofrat Prof. Dr. F. Kö n i g für die Überlassung der Arbeit meinen verbindlichsten Lank auszusprechen.

\section{Literaturverzeichnis.}

I. Asch of $f$, Pathologische Anatomie.

2. Brunzel. Deutsche Zeitschr. f. Chir., Bd. 145, H. I-2.

3. Doebler, Über Invaginatio gastroduodenalis. Inaug.-Diss. Breslau 1918.

4. Doose, Der EinfluB der Kriegskost auf Brucheinklemmung und mechanischen Ileus. Deutsche med. Wochenschr. 1917, 46.

5. Grünfeld, Ein Fall von Ileus während der Schwangerschalt. Inaug.Diss. Erlangen 1918. 
6. H o che negg, Lehrbuch der speziellen Chirurgie.

7. Hofmann, Krieg und chirurgische Erkrankungen der Zivilbevölkerung. Münchner med. Wochenschr. 1917, 35.

8. Ka us ch, Pathologie und Therapie des Ileus.

9. König, Lehrbuch der Chirurgie.

ı. -, Eingeweidebrüche im Krieg. Deutsche med. Wochenschr. 1917, 1.

I I. - Über Ileus. Münchner med. Wochenschr. I9I7, 39.

12. Spasukosky, Volvulus intest. als Krankheit der hungernden Menschen. Arch. f. klin. Chir. 1909, Bd. gr.

13. Stransky, Krieg und Bevölkerung. Wiener klin. Wochenschr. I9i6, $15-17$.

14. T a n d ler, Krieg und Bevölkerung.

15. Treder, Ein Fall von Ileus bei Coecum mobile. Inaug.-Diss. Berlin 1918.

16. W i e $m$ a $n n$, Uber Brucheinklemmung und Kriegsernährung. Deutsche Zeitschr. f. Chir. 1917, I40.

17. Wilms, Der Ileus. Deutsche Chir. 1906, Lfg. $46 \mathrm{~g}$.

18. Z untz, Einfluß des Krieges auf Ernährung und Gesundheit des deutschen Volkes. Med. Klinik 1915. 